\title{
Iris cyst after femtosecond laser-assisted cataract surgery: a case report
}

\author{
Po-Ying $\mathrm{Wu}^{1}$, Meng-Hsien $\mathrm{Wu}^{2,3}$, Chi-Cheng $\mathrm{Wu}^{3}$ and Chi-Chin Sun ${ }^{4,5^{*}}$
}

\begin{abstract}
Background: Secondary iris cysts are uncommon complication after cataract surgery. The reports of an iris cyst after conventional phacoemulsification surgery are scanty, let alone the iris cyst following femtosecond laserassisted cataract surgery (FLACS). We herein report an unusual case of an iris cyst after an uneventful FLACS.

Case presentation: A 64-year-old man who was healthy underwent FLACS for a moderate cataract of his left eye. Shortly after surgery, he achieved 20/20 vision, but anterior bowing of temporal iris was noted on postoperative day 9 with a retro-pupillary iris cyst at temporal-inferior quadrant found after pupil dilatation. The cyst was confirmed by ultrasound bio-microscopy afterward. Four weeks later, argon laser cystotomy was performed, and the cyst disappeared 3 days later. The patient's vision remained stable thereafter.
\end{abstract}

Conclusion: Although rare, secondary iris cyst may be one of the complications after FLACS. Argon laser cystotomy is effective in the management of post-FLACS iris cyst.

Keywords: Iris cyst, Femtosecond laser-assisted cataract surgery (FLACS), Argon laser cystotomy, Case report

\section{Background}

Iris cysts are uncommon and can be classified into two categories, primary and secondary, while secondary iris cysts are even less common than primary cysts [1]. Secondary cysts can be implantation cysts, originating by an invasion of conjunctival or corneal epithelial cells following surgical trauma or a penetrating wound [2]. These secondary iris cysts are thought to be the complications of large corneal incisions during surgeries, for instance, extra-capsular cataract extraction (ECCE), intra-capsular cataract extraction with vitreous loss $(\mathrm{ICCE}+\mathrm{VL})$, and penetrating keratoplasty [3].

Femtosecond laser-assisted cataract surgery (FLACS) has been widely used nowadays since it first introduced in 2008 [4]. The advantages of FLACS over

\footnotetext{
* Correspondence: arvin.sun@msa.hinet.net

${ }^{4}$ Department of Medicine, College of Medicine, Chang Gung University, Taoyuan, Taiwan

${ }^{5}$ Department of Ophthalmology, Chang Gung Memorial Hospital, 222 Mai Chin Road, An Leh District, Keelung, Taiwan

Full list of author information is available at the end of the article
}

conventional phacoemulsification surgery (CPE) are its precision and predictability. Although FLACS is thought to be a safe and trustable technique, it can still cause several post-operative complications, such as elevated intraocular pressure and macular edema [5]. Nevertheless, there is no report of secondary iris cyst after FLACS. Even in CPE, only 2 cases of iris cyst following CPE have been reported [6,7]. The iris cysts in the two cases were found 2 months and 3 years postoperatively, respectively. Herein, we report an unusual case of an iris cyst presented shortly after an uneventful FLACS and was successfully treated by argon laser cystotomy.

\section{Case presentation}

A 64-year-old man who was otherwise healthy underwent FLACS for a moderate cataract in his left eye due to the rapidly worsening of vision during outdoor and night driving. Preoperative ocular examination showed moderate nuclear sclerotic and posterior subcapsular cataract, relatively shallow anterior chamber depth 
(2.33 mm) with corrected distance visual acuity (CDVA) of $20 / 60$ in the left eye and no other ocular pathology was noted.

Uneventful FLACS was performed using LenSx (Alcon, Fort Worth, TX, USA) with regular setting of circular continuous capsulorrhexis, fragmentation, and wound construction. Phacoemulsification with temporal approach using Centurion (Alcon, Fort Worth, TX, USA) was then carried out smoothly as routine surgery. Postoperative care and follow-up were unremarkable, and he gained uncorrected visual acuity of $18 / 20$ on postoperative day one.

However, anterior bowing of iris and asymmetrically shallowing of the anterior chamber were noted on the temporal side without any visual disturbance 9 days after operation. An iris mass was found after pupil dilatation (Fig. 1a,b), but there was no other ocular abnormality and the intraocular lens remained in situ. A retropupillary iris cyst was identified by ultrasound biomicroscopy (Fig. 2a).

Despite the iris cyst, the postoperative CDVA of the left eye remained 20/20 during the follow-up period. Owing to not obvious cyst regression, argon laser cystotomy (50 um/0.2 sec / $500 \mathrm{~mW} / 16$ shots) was applied under the slit lamp one month later. The iris cyst became smaller 3 days after the procedure (Fig. 1c). Repeated ultrasound bio-microscopy demonstrated no residual iris cyst (Fig. 2b). The CDVA in his left eye was $20 / 20$ and remained stable after cystotomy.

\section{Discussion}

The pathogenesis of secondary cysts is that conjunctival or corneal epithelial cells invade into the anterior chamber following surgical trauma or a penetrating wound [2]. Iris, a vascularized tissue with rich nutrients, provides epithelial cells an ideal place for proliferation, resulting in an iris cyst. Histopathology of secondary implantation cysts is composed of multiple layers of non-keratinized stratified squamous epithelium [8]. Symptoms or signs of secondary iris cysts are varied. Iritis or uveitis is found to be the most common symptoms. A history of preceding surgery, trauma, or inflammation is nearly always present. For those iris cysts covering the visual axis, visual disturbance can also be the chief complaint of the patients.

It is possible that epithelial cells may be carried to anterior chamber by surgical instruments. This can explain why post-operative iris cysts have not been reported previously in FLACS owing to a corneal incision in FLACS created by femtosecond laser rather than a surgical instrument. Since formation of secondary iris cysts relates to epithelial downgrowth, the iris cysts should be uncommon in both FLACS and CPE due to small corneal incisions, theoretically. As a result, it is not surprising that the rare cases of iris cysts were reported after CPE $[6,7]$, let alone the report in FLACS postoperatively. One of the advantages of FLACS is that wound length created by femtosecond laser is also comparably small and precise as those created by keratomes. However, using femtosecond lasers can create more ideal clear
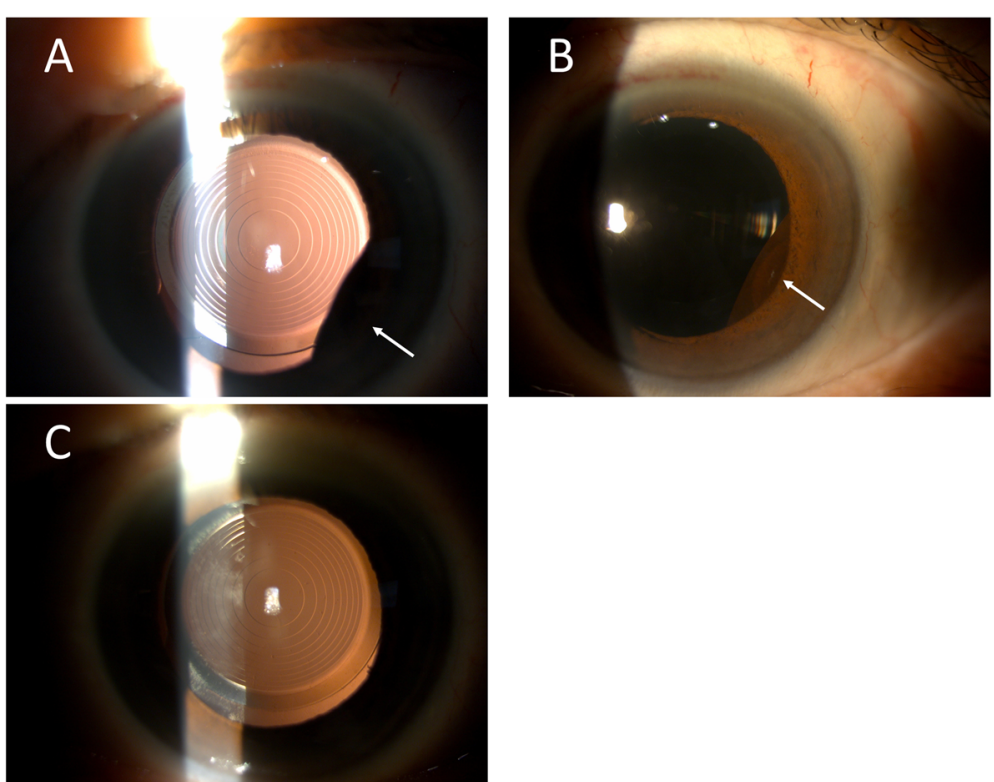

Fig. 1 External eye photographs of the left eye in the 64-year-old patient. a, b A retro-pupillary iris mass at temporal-inferior quadrant (white arrows) was found after pupil dilatation nine days after FLACS. c The iris cyst disappeared three days after argon laser cystotomy, and the intraocular lens was still in good position 

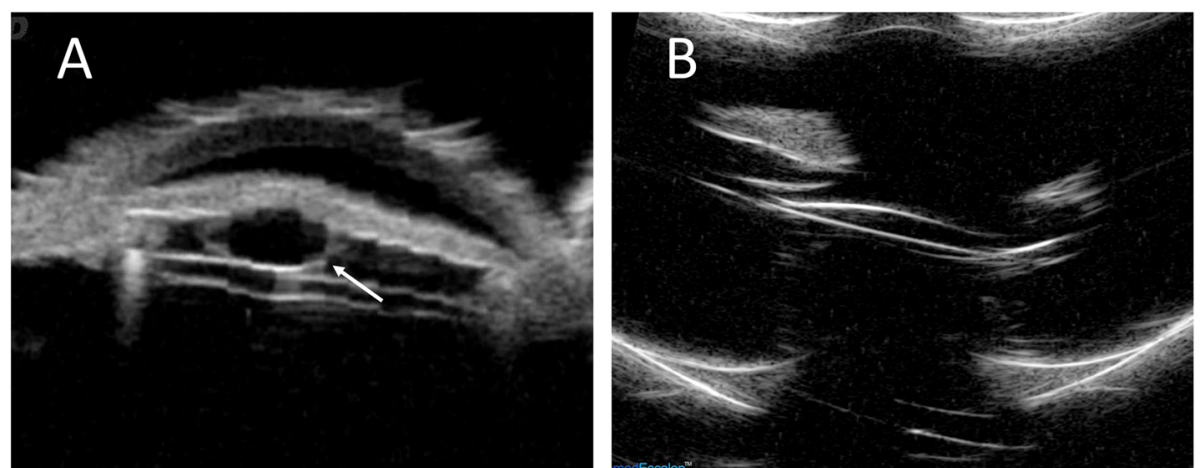

Fig. 2 Ultrasound bio-microscopic images of the left eye in the 64-year-old patient. a Ultrasound bio-microscopy showed a typical hallow retropupillary cyst (white arrow). b Repeated ultrasound bio-microscopy confirmed no residual iris cysts 10 days after argon laser cystotomy

corneal incisions with lower rates of endothelial gap, endothelial misalignment, and Descemet membrane detachment compared with those using metal keratomes [9]. This may also explain the low incidence of secondary iris cysts after FLACS.

However, adopting FLACS does not guarantee the freedom from iris cysts. We hypothesize that a subtle injury to iris during femtosecond laser application, subsequent phacoemulsification or any other steps might provide an adequate vascularized space for epithelial cells to colonize and proliferate. For example, iris injury may be induced during irrigation and aspiration. In either FLACS or CPE, this crucial step cannot be avoided. Previous study indicated that FLACS can reduce cumulative dissipated energy, which may result in the lower risk of anterior chamber inflammation [10]. We infer that less ultrasound energy may also reduce the damage to iris. Nevertheless, femtosecond laser pulse may directly harm the iris if the built-in positioning system does not properly work. Further studies are mandatory to investigate the influence of ultrasound or laser energy to iris or compare the damage to iris in FLACS and CPE.

It is also interesting that the onset of the iris cyst in our patient was only 9 days after FLACS. Other cases of a secondary iris cyst usually reported as late-onset from months to years after the surgery [2]. It could be explained by the easy accessibility of medical services in our country. Unlike other cases, our patient was found in the regular postoperative examination on day 9 without any discomfort or visual disturbance. We think that iris cysts in other cases may be appeared shortly but were not discovered until the occurrence of any symptoms.

Treatments for iris cyst include simple observation, fine-needle aspiration, laser (including argon or Nd:YAG laser), or surgical excision. Laser therapy has become the current trend because of minimal invasion. Secondary iris cysts possess a greater potential for associated complications compared with primary iris cysts [2]. For example, the coincidence of the phakic intraocular lens and the cyst may lead to potentially sight-threatening complications [11]. Argon laser cystotomy appears to provide an effective result for postoperative iris cysts [12]. Though we successfully applied the argon laser cystotomy to resolve the iris cyst after FLACS, however, long term follow-up is needed to observe possible complications of laser cystotomy including focal lens or corneal damage, retinal detachment, bleeding, elevated intraocular pressure, and progression of peripheral anterior synechia.

In conclusion, though FLACS is a safe and precise technique, pre-programmed corneal incisions in FLACS does not guarantee the freedom from iris cysts. Clinicians should carefully inspect the patients after FLACS since an iris cyst may be asymptomatic and shortly occur after surgery. Argon laser cystotomy is an effective tool for managing post-FLACS iris cyst.

\section{Abbreviations}

FLACS: Femtosecond laser-assisted cataract surgery; ECCE: Extra-capsular cataract extraction; ICCE + VL: Intra-capsular cataract extraction with vitreous loss; CPE: Conventional phacoemulsification surgery; CDVA: Corrected distance visual acuity

\section{Acknowledgements}

Not applicable.

\section{Authors' contributions}

PYW, MHW, and CCW took primary care of the patient. MHW and CCS designed the current study. PYW, MHW and CCS drafted the work. CCW analyzed the data. CCS substantially revised the work. All authors read and approved the final manuscript.

\section{Authors' information (optional)}

Not applicable.

\section{Funding}

The authors declare that they have no additional funding support.

Availability of data and materials

The datasets used and/or analyzed during the current study are available from the corresponding author on reasonable request. 


\section{Ethics approval and consent to participate}

The study was approved by the Institutional Review Board of Chang Gung Memorial Hospital (Approval No:: 202000658B0) and followed the tenets of the Declaration of Helsinki.

\section{Consent for publication}

The written informed consent to publish this information was obtained from study participants and the proof of consent can be requested at any time.

\section{Competing interests}

The authors declare that they have no competing interests 140 .

\section{Author details}

'Department of Medicine, College of Medicine, National Taiwan University, Taipei, Taiwan. ${ }^{2}$ Cheng-Ching Eye Institute, Kaohsiung, Taiwan. ${ }^{3}$ Department of Business Management, National Sun Yat-sen University, Kaohsiung, Taiwan. ${ }^{4}$ Department of Medicine, College of Medicine, Chang Gung University, Taoyuan, Taiwan. ${ }^{5}$ Department of Ophthalmology, Chang Gung Memoria Hospital, 222 Mai Chin Road, An Leh District, Keelung, Taiwan.

Received: 21 April 2020 Accepted: 4 January 2021

Published online: 13 January 2021

\section{References}

Shields JA. Primary cysts of the iris. Trans Am Ophthalmol Soc. 1981;79: 771-809.

1.

Georgalas I, Petrou P, Papaconstantinou D, Brouzas D, Koutsandrea C, Kanakis M. Iris cysts: A comprehensive review on diagnosis and treatment.

2. Surv Ophthalmol. 2018;63(3):347-64.

Behrouzi Z, Khodadoust A. Epithelial iris cyst treatment with intracystic ethanol irrigation. Ophthalmology. 2003;110(8):1601-5.

Agarwal A, Jacob S. Current and effective advantages of femto phacoemulsification. Curr Opin Ophthalmol. 2017;28(1):49-57.

Ye Z, Li Z, He SA, Meta-Analysis. Comparing Postoperative Complications and Outcomes of Femtosecond Laser-Assisted Cataract Surgery versus

5. Conventional Phacoemulsification for Cataract. J Ophthalmol. 2017;2017:

3849152.

Kuganasan S, Voon Loo A, Subrayan V. A rare occurrence of epithelial inclusion iris cyst after phacoemulsification. Clin Exp Optom. 2015;98(1):97-8.

6.

Shah A, Rajesh PS, Dutta Majumder P. Iris cyst following cataract surgery. Clin Exp Optom. 2019.

Rishi P, Rishi E, Biswas J, Nandi K. Clinical and histopathological features of posttraumatic iris cyst. Indian J Ophthalmol. 2008;56(6):518-21.

Grewal DS, Basti S. Comparison of morphologic features of clear corneal incisions created with a femtosecond laser or a keratome. J Cataract Refract

9. Surg. 2014;40(4):521-30.

Kolb CM, Shajari M, Mathys L, Herrmann E, Petermann K, Mayer WJ, et al.

Comparison of femtosecond laser-assisted cataract surgery and

10. conventional cataract surgery: a meta-analysis and systematic review. J Cataract Refract Surg. 2020;46(8):1075-85.

Gharaibeh AM, Liehneova I, Mojzis P, Ziak P, Alio JL. Iris and ciliary body cysts and phakic intraocular lenses. Eur J Ophthalmol. 2019:

11. 1120672119876532 .

Scholz RT, Kelley JS. Argon laser photocoagulation treatment of iris cysts following penetrating keratoplasty. Arch Ophthalmol. 1982;100(6):926-7.

12

\section{Publisher's Note}

Springer Nature remains neutral with regard to jurisdictional claims in published maps and institutional affiliations.

Ready to submit your research? Choose BMC and benefit from:

- fast, convenient online submission

- thorough peer review by experienced researchers in your field

- rapid publication on acceptance

- support for research data, including large and complex data types

- gold Open Access which fosters wider collaboration and increased citations

- maximum visibility for your research: over $100 \mathrm{M}$ website views per year

At BMC, research is always in progress.

Learn more biomedcentral.com/submissions 